\section{Tratamento homeopático de crianças com úlcera de córnea em escudo por ceratoconjuntivite primaveril: relato de casos e aspectos bioéticos}

\section{Homeopathic treatment of children with shield ulcers of the cornea in vernal keratoconjunctivitis: case reports and bioethical dimensions}

Antônio Carlos Gonçalves da Cruz 1

Cláudio Maciel Sena 2

Marco Antônio Guarino Tanure 3

Joel Edmur Boteon 4

Elza Machado de Melo 5

Programa de Doutoramento em Bioética. Faculdade de Medicina. Universidade do Porto. Alameda Prof. Hernani Monteiro, 4200-319. Porto, Portugal. E-mail: imh@imh.com.br

2 Programa de Doutorado em Ciências Aplicada à Cirurgia e Oftalmologia. Universidade Federal de Minas Gerais. Belo Horizonte, MG, Brasil.

3 Departamento de Córnea. Hospital São Geraldo. Universidade Federal de Minas Gerais. Belo Horizonte, MG, Brasil.

4 Departamento de Oftalmologia e Otorrinolaringologia. Universidade Federal de Minas Gerais. Belo Horizonte, MG, Brasil.

5 Departamento de Medicina Preventiva e Social. Universidade Federal de Minas Gerais. Belo Horizonte, MG, Brasil.

\section{Abstract}

Objectives: present a homeopathic treatment of corneal ulcers for vernal keratoconjunctivitis and its bioethical implications.

Methods: nine cases were studied, after having been referred following the failure of conventional treatments. Medications were chosen according to the specific characteristics of the patients, including psychiatric disorders. The medication costs were gathered by consulting three homeopathic and three non-homeopathic pharmacies in Belo Horizonte. The estimate for conventional treatment cost was based on the medication prescribed for the patient prior to the commencement of homeopathic care.

Results: scarring occurred between fifteen and one-hundred eighty days, with an average medication cost of eight dollars, roughly one-thirteenth of the estimated direct cost of one month of conventional treatment. The similarity of therapeutic approaches should minimize bioethical concerns regarding the medical care of children with vernal keratoconjunctivitis.

Conclusions: homeopathy can contribute to both the medical and bioethical aspects of treatment of this disease.

Key words Conjunctivitis allergic, Homeopathy, Law of similars, Bioethics, Human experimentation

\section{Resumo}

Objetivos: apresentar um tratamento homeopático de úlcera de córnea por ceratoconjuntivite primaveril, considerando-o bioeticamente.

Métodos: foram usados nove casos, encaminhados por insucesso terapêutico convencional. Escolheram-se os medicamentos de acordo com caracteristicas singularizantes dos pacientes que incluiram o psiquismo. Levantaram-se os custos medicamentosos consultando-se três farmácias homeopáticas e três não-homeopáticas de Belo Horizonte. O tratamento convencional foi estimado a partir da medicação usada pelos enfermos antes do início dos cuidados homeopáticos.

Resultados: as cicatrizações ocorreram entre quinze e cento e oitenta dias, com custo medicamentoso médio de oito dólares, cerca de treze vezes menor do que a estimativa de custo direto de um mês da abordagem convencional. O manejo terapêutico da similitude pode minimizar preocupações bioéticas relativas à atenção à saúde de crianças com ceratoconjuntivite primaveril.

Conclusões: a homeopatia pode colaborar para o tratamento desta enfermidade, aprimorando-o bioeticamente.

Palavras-chave Conjuntivite alérgica, Homeopatia, Lei dos semelhantes, Bioética, Experimentação humana 


\section{Introdução}

A ceratoconjuntivite primaveril é uma afecção alérgica severa, recorrente e bilateral. Caracteriza-se por uma inflamação conjuntival grave, que frequentemente acomete a córnea, ${ }^{1}$ ameaçando a visão. ${ }^{2}$ Ocorre em grande parte do mundo, ${ }^{1}$ sendo comumente observada em crianças e adultos jovens. Há predominância no sexo masculino, com uma incidência de aproximadamente 3:1 até a puberdade. Após esse período, homens e mulheres são igualmente acometidos. ${ }^{1}$ Os pacientes geralmente apresentam prurido, lacrimejamento, fotofobia e sensação de corpo estranho. Estes sintomas se exacerbam na primavera e no verão, mas podem permanecer por todo o ano devido a sua natureza crônica. ${ }^{3}$ Nos casos mais severos são comuns interferência e interrupção nas atividades diárias do paciente. 2 Biomicroscopicamente, encontram-se hiperemia da conjuntiva bulbar, hipertrofia papilar da conjuntiva tarsal, nódulos de Trantas, edema límbico e secreção mucosa. ${ }^{3} \mathrm{O}$ comprometimento corneano se inicia com erosões epiteliais puntiformes, causadas pelos mediadores liberados pela conjuntiva e exacerbada pelo trauma mecânico das papilas gigantes sobre a córnea. ${ }^{4}$ Pode haver coalescência dessas erosões, resultando em um defeito epitelial conhecido como úlcera em escudo. ${ }^{4}$ Deve-se lembrar que pacientes com úlcera de córnea em escudo necessitam de tratamento com corticóides tópicos em altas doses por curto período de tempo, 4 os quais nem sempre são eficazes e podem causar glaucoma, formação de catarata $^{2}$ e infecções oculares secundárias. ${ }^{5}$ Assim, tem sido empregada, no tratamento desta doença a medicina homeopática que se apóia no princípio denominado semelhança, na experimentação na saúde humana e no manejo de doses infinitesimalmente diluídas. ${ }^{6-9}$

Foi a partir do final do século XVIII que Hahnemann a sistematizou, dedicando-se a experimentações de ultradiluições medicamentosas em sua própria saúde e na de alguns voluntários. Seu propósito era catalogar as perturbações na saúde decorrentes de cada prova e, assim, estruturar um registro de memórias experimentais - matéria médica - a fim de a ele recorrer, mediante recordação ou reconhecimento, para aplicar terapeuticamente o princípio de similitude. Uma matéria médica encerra, então, o que se conhece como memórias experimentais, mais propriamente, memórias sintéticas experimentais de semelhança. ${ }^{10}$

No Brasil, a homeopatia foi reconhecida como especialidade médica pelo Conselho Federal de Medicina em 198011 e enfoca o indivíduo como um todo e não somente as doenças. Devido a essa individualização, são imprescindíveis diferentes medicamentos para tratar pacientes com a mesma doença. Conseguintemente, podem-se reproduzir resultados de melhora na ceratoconjutivite primaveril utilizando-se medicamentos homeopáticos individualizados para cada paciente. $1,9,10$

Com o extraordinário progresso tecnológico decorrente do impacto mecanicista sobre a natureza e o humano, graves preocupações com a sobrevivência e com a qualidade de vida das pessoas, no âmbito dos direitos do Homem, desencadearam movimentos bioéticos em busca de diálogo éticotécnico no terreno da multi, inter e transdisciplinaridade dos saberes teórico-práticos. ${ }^{12,13}$ No cerne desses problemas encontram-se os custos elevados e em constante elevação com a atenção à saúde, em face de sua dependência da biotecnologia.

Alternativas terapêuticas que concorram para minimizar os custos da biomedicina, desde a convenção da Organização Mundial da Saúde de Alma-Ata (1978), como a medicina homeopática, têm sido estimuladas a colaborar para o saneamento de injustiças e de iniquidades no uso dos recursos para a atenção à saúde. 14,15

Embora se possa considerar que a homeopatia não convenha para reduzir custos de tratamentos, em face da complexidade que envolve o grau de comprometimento do organismo pelas enfermidades e das avaliações econômicas, 16 frequentemente se aceita que seu custo medicamentoso é mais baixo do que o da medicina convencional.9,16

A medicina homeopática singulariza os casos de perturbações de saúde para tratar as representações de doenças, 1,9 levando-se em conta que ela compreende as enfermidades clínicas como acidentes que particularizam sensorial e materialmente a totalidade da saúde individual alterada. $6,7 \mathrm{O}$ uso de diferentes medicamentos homeopáticos para tratar os pacientes com úlcera de córnea em escudo se faz necessário porque para a homeopatia a doença não ocorre de modo separado do conjunto vivo do organismo, ou seja, é o organismo como uma generalidade singular que adoece e não apenas uma parte dela.6,7 Por isso, com a medicina homeopática, é indispensável manejar a totalidade sintomática que individualiza o enfermo para tratar qualquer alteração de saúde (ocular ou de outro sistema) do paciente, a fim de se conseguir boa resposta. Singularizam-se os casos através da inclusão de diferenças que os enfermos apresentam, ainda que portadores de uma mesma patologia.

Assim o presente trabalho objetiva apresentar nove casos de tratamento homeopático de crianças 
portadoras de ceratoconjuntivite primaveril com úlcera de córnea em escudo, considerando-os bioeticamente, com ênfase nos princípios da nãomaleficência, da justiça e da autonomia..

\section{Métodos}

Estudaram-se nove prontuários de pacientes que foram encaminhados ao Hospital São Geraldo da UFMG, a fim de participarem da pesquisa denominada "Uso da Medicação Homeopática no Tratamento da Ceratoconjuntivite Primaveril", provenientes de outros serviços em razão de insucesso pela abordagem ortodoxa. Os enfermos eram do sexo masculino e portadores de ceratoconjuntivite primaveril com úlcera de córnea em escudo uni ou bilateral (Tabela 1). Todos foram examinados no Departamento de Córnea e Doenças Externas da referida instituição e também apresentavam história de broncoespasmo. A idade média dos pacientes foi de sete anos (de 4 a 11 anos). Antes do tratamento homeopático, estavam eles em uso de estabilizador de mastócitos, corticóide, colírio lubrificante e reparador oftálmico, sendo que alguns utilizavam ainda imunossupressor. Entretanto, somente o paciente 2 apresentava opacificação subcapsular posterior em ambos os olhos (AO) e glaucoma cortisônico, controlado com o uso de latanoprost $0,005 \%$ e maleato de timolol $0,5 \%$ em AO.

Para iniciar e conduzir o tratamento homeopático, foram suspensas todas as medicações convencionais tópicas e sistêmicas, até que se esgotasse sua ação paliativa, exceto em relação a um paciente (caso 2), devido ao glaucoma cortisônico em ambos os olhos. Neste caso, introduziu-se a abordagem homeopática concomitantemente à redução do corticóide sistêmico, porém manteve-se a medicação antiglaucomatosa (latanoprost $0,005 \%$ e maleato de timolol 0,5\%). Desde a descontinuação do uso convencional de medicamentos ao começo do

\section{Tabela 1}

Idade, lateralidade da úlcera, medicação homeopática, memória sintética experimental e doses.

\begin{tabular}{|c|c|c|c|c|c|}
\hline Casos & $\begin{array}{l}\text { Idade } \\
\text { (anos) }\end{array}$ & $\begin{array}{c}\text { Úlcera } \\
\text { em escudo }\end{array}$ & Medicamento & $\begin{array}{l}\text { Memória sintética } \\
\text { experimental }\end{array}$ & $\begin{array}{l}\text { Número de doses do } \\
\text { medicamento }\end{array}$ \\
\hline 1 & 6 & bilateral & Kalium carbonicum & $\begin{array}{l}\text { sono sobressaltado, medo quando sozinho } \\
\text { a noite e grande irritabilidade }\end{array}$ & 1 \\
\hline 2 & 9 & bilateral & Vaccininum & $\begin{array}{l}\text { psiquismo insatisfeito e irritado e cronici- } \\
\text { dade do quadro com iatrogenias }\end{array}$ & 2 \\
\hline 3 & 7 & unilateral & Natrum sulphuricum & $\begin{array}{l}\text { sobressalto por ruídos, aversão a conversar } \\
\text { e agrava por umidade }\end{array}$ & 2 \\
\hline 4 & 7 & unilateral & Arsenicum album & inquietude, ansiedade pela cura e ardências & 1 \\
\hline 5 & 4 & bilateral & Baryta carbonica & $\begin{array}{l}\text { lentidão para compreensão, timidez e } \\
\text { friorento }\end{array}$ & 2 \\
\hline 6 & 5 & unilateral & Phosphorus & $\begin{array}{l}\text { medo de escuro e de morte, preocupação } \\
\text { sobre doenças em familiares e sofrimentos } \\
\text { por desamparo, imaginários ou não, } \\
\text { incluindo febres }\end{array}$ & 1 \\
\hline 7 & 11 & unilateral & Pulsatilla & submisso, suave e melhora ao ar livre & 1 \\
\hline 8 & 8 & unilateral & Bryonia & $\begin{array}{l}\text { deseja quietude, muita sede e obstipação } \\
\text { crônica }\end{array}$ & 1 \\
\hline 9 & 7 & unilateral & Apis mellifica & $\begin{array}{l}\text { gritos noturnos, agravamento pelo calor e } \\
\text { ciúmes }\end{array}$ & 1 \\
\hline
\end{tabular}


tratamento homeopático, todos os pacientes foram acompanhados com retornos frequentes, às vezes diários, de acordo com a gravidade dos sinais e sintomas oculares.

O tratamento homeopático foi realizado com medicamentos peculiares, ${ }^{17}$ preparados na escala centesimal hahnemanniana em doses (ultra-diluições ou diluições infinitesimais), via oral, baseando-se na totalidade sintomática individualizante do paciente, de que resulta a necessidade de respeito aos aspectos individuais de cada enfermo em suas peculiaridades dinâmicas de adoecimento, incluindo o seu psiquismo (Tabela 1).

A escolha dos medicamentos similares se deu no âmbito do Serviço Phýsis do Instituto Mineiro de Homeopatia, guiada por indicações contidas na matéria médica clínica homeopática. 17

Exceto no caso 2, permitiu-se somente o uso tópico de lubrificantes oculares (colírio ou gel) e de solução de cloreto de sódio a $0,9 \%$. Neste paciente fez-se um desbridamento em uma ulceração no olho direito (OD), utilizando-se lâmina número $15 \mathrm{e}$ oclusão com um reparador oftálmico em pomada (aminoácido $25 \mathrm{mg}$, metionina $5 \mathrm{mg}$, acetato de retinol $10.000 \mathrm{UI}$ e cloranfenicol $5 \mathrm{mg}$ ) por dois dias.

Durante o período de tratamento, as intercorrências oculares ou sistêmicas foram conduzidas pelo médico homeopata, a não ser no caso do paciente 2 , quanto à intervenção oftalmológica para desbridamento da ulceração no OD.

Os custos médios medicamentosos homeopático e convencional foram levantados por uma pesquisa de preços praticados em farmácias centrais da cidade de Belo Horizonte: três homeopáticas e três nãohomeopáticas. $\mathrm{O}$ do tratamento convencional foi calculado a partir da medicação usada pelos pacientes antes do início do tratamento, considerando-se a inclusão de imunossupressor (ciclosporina) para se estimar o custo medicamentoso máximo, já que nem todos o usavam. A medicação antiglaucomatosa não foi incluída nesta estimativa.

Este trabalho foi aprovado pelo Comitê de Ética em Pesquisa da Universidade Federal de Minas Gerais (UFMG) através do Parecer n ETIC 158/99.

\section{Resultados}

As curas se deram entre quinze e cento e oitenta dias

Tabela 2

Tamanho da úlcera e tempo de cicatrização.

\begin{tabular}{|c|c|c|c|}
\hline Casos & Olho afetado & Tamanho da úlcera $(\mathrm{mm})$ & Tempo para cicatrização da úlcera (dias) \\
\hline \multirow[t]{2}{*}{1} & direito & 2,0 & 30 \\
\hline & esquerdo & 1,5 & 15 \\
\hline \multirow[t]{2}{*}{2} & direito & 0,5 & 15 \\
\hline & esquerdo & $0,5 \times 2,0$ & $60 *$ \\
\hline \multirow[t]{2}{*}{3} & direito & sem úlcera & - \\
\hline & esquerdo & $2,0 \times 2,5$ & 30 \\
\hline \multirow[t]{2}{*}{4} & direito & $0,8 \times 0,8$ & 150 \\
\hline & esquerdo & sem úlcera & - \\
\hline \multirow[t]{2}{*}{5} & direito & $3,0 \times 4,0$ & 60 \\
\hline & esquerdo & $8,0 \times 4,0$ & 180 \\
\hline \multirow[t]{2}{*}{6} & direito & sem úlcera & - \\
\hline & esquerdo & $0,7 \times 0,7$ & 30 \\
\hline \multirow[t]{2}{*}{7} & direito & $2,0 \times 2,0$ & 30 \\
\hline & esquerdo & sem úlcera & - \\
\hline \multirow[t]{2}{*}{8} & direito & sem úlcera & - \\
\hline & esquerdo & $1,4 \times 1,0$ & 60 \\
\hline \multirow[t]{2}{*}{9} & direito & sem úlcera & - \\
\hline & esquerdo & $2,0 \times 2,5$ & 120 \\
\hline
\end{tabular}

*Foi realizado desbridamento da úlcera com lâmina número 15. 
(Tabela 2) e em todos os casos a cicatrização da ulceração corneana foi precedida pelo alívio dos sintomas particulares e gerais.

No caso 1 , decorridos três meses da cicatrização da lesão do olho esquerdo (OE), surgiu uma úlcera de córnea no olho direito (OD), que foi diagnosticada em um exame de rotina. Após 15 dias, a córnea do OD estava totalmente epitelizada.

No caso 2, depois da cicatrização da úlcera do $\mathrm{OD}$, foi realizado um desbridamento da úlcera no OE, seguido por oclusão, com um reparador oftálmico em pomada por dois dias, indicado pelo oftalmologista com o objetivo de acelerar a cicatrização.

No caso 3, concomitantemente com a melhora dos sintomas oculares, ocorreu um aumento de aproximadamente três vezes do tamanho inicial da úlcera de córnea em escudo do OE. Nesse mesmo período, foi prescrito um lubrificante ocular em gel três vezes ao dia. Depois deste aumento, a lesão evoluiu com uma cicatrização progressiva até a cura, ocorrida em um mês.

Nos pacientes 4, 6, 7, 8 e 9, a úlcera de córnea em escudo cicatrizou sem nenhuma intercorrência, em períodos variados (Tabela 2 ).

No caso 5, a úlcera do OD cicatrizou em dois meses e no OE, somente ao fim de seis meses.

Não foi necessário nenhum outro tipo de intervenção medicamentosa para tratamento de intercorrências como crises de broncoespasmo (casos 1 e 5), infecções de vias aéreas superiores (casos 1, 2, 5), erupções cutâneas (casos 3 e 5) e de diarréia (caso 7).

O número médio de doses medicamentosas homeopáticas foi de 1,3 por paciente, já que três pacientes usaram duas doses e os demais, uma (Tabela 1), correspondendo ao custo atual de oito dólares. Assim, o custo médio mensal do tratamento medicamentoso homeopático foi avaliado em um dólar e trinta e quatro centavos, levando-se em conta que a cura mais demorada se deu em seis meses.

Estimou-se o custo medicamentoso convenciona mensal e médio em cento e oito dólares: noventa e dois dólares no caso de não inclusão de imunossupressor e cento e vinte e quatro no caso contrário.

Todas as crianças se reintegraram às suas atividades habituais antes ou paralelamente (nas curas em poucas semanas) à cicatrização corneana, em concomitância com a melhora objetiva e subjetiva de seus sintomas singulares.

Perceberam-se frequentes manifestações de satisfação dos familiares das crianças desde a fase de melhora sintomática, mesmo antes da cicatrização corneana.

\section{Discussão}

As cicatrizações das lesões corneanas podem ter sido naturais, sobretudo nas evoluções mais tardias. Porém, o alívio dos sintomas antes delas sugere que as curas se deram em decorrência dos cuidados homeopáticos que contribuem para harmonização dinâmica do organismo desde seu interior, anteriormente à erradicação das atualizações locais da enfermidade.6,7 As curas mais tardias podem sugerir evolução natural, mas a melhora da representação de totalidade singular aponta para resposta ao tratamento. Por outro lado, as crianças foram encaminhadas para a abordagem homeopática porque não se obtinha êxito com a intervenção ortodoxa, já que todas elas eram cuidadas pela convenção, tendo, inclusive, o paciente dois, desenvolvido opacificação subcapsular posterior e glaucoma cortisônicos.

Os portadores de ceratoconjuntivite primaveril severa apresentam risco maior de redução da acuidade visual 2 devido às complicações corneanas e aos efeitos colaterais dos corticóides. Esta situação, aliada ao impacto gerado pelas despesas com o tratamento medicamentoso convencional, desencadeia problemas bioéticos nos campos da nãomaleficência e da justiça social. Enquanto o custo medicamentoso médio e mensal do tratamento ortodoxo situou-se entre noventa e dois e cento e vinte e quatro dólares, o custo médio de todo o tratamento medicamentoso homeopático aproximou-se de oito dólares. Considerando-se que a estimativa de custo medicamentoso mensal e médio do tratamento aproximou-se de um dólar e trinta e quatro centavos, pode-se ampliar as reflexões sobre a interferência no consumo de recursos para os cuidados com a úlcera de córnea em escudo por ceratoconjuntivite primaveril baseados em uma terapêutica homeopática.

A paliação da ceratoconjuntivite primaveril se associa à não inclusão de co-morbidades a esta doença como manifestações de uma mesma enfermidade que acometa a totalidade singular do organismo. Enquanto o uso paliativo de drogas convencionais tende a se implicar com a iatrogenia e, consequentemente, com a ampliação de gastos com a atenção aos portadores de úlcera de córnea por ceratoconjuntivite primaveril, a medicina homeopática tende a totalizar a diversidade mórbida como manifestações de uma mesma enfermidade, susceptível de ser tratada simplificadamente por um mesmo medicamento individualizado, semelhante suave, voltando-se para a maximização da economia nos cuidados com os pacientes em questão. 18

Em um processo de cura homeopática é funda- 
mental não interferir enquanto o medicamento selecionado estiver agindo. Isso é importante para se obter o mais alto ideal da cura, que é o restabelecimento rápido, suave e duradouro da saúde pelo caminho mais curto, mais seguro e menos prejudicial para o paciente, de forma individualizada. ${ }^{6,7} \mathrm{Tal}$ fato pode ser constatado nos casos 1,2 e 4 . No primeiro a não interferência na lesão nova (úlcera de córnea assintomática no OD) levou à epitelização rápida sem sofrimento para a criança. No caso 2,0 desbridamento da úlcera de córnea, na tentativa de aceleração do processo de cura, apesar da boa evolução homeopática, se associou a uma agravação dos sinais e sintomas a despeito da rápida cicatrização da úlcera. No caso 4, verificou-se uma lesão relativamente pequena, mas com um período de cicatrização muito longo (cinco meses). Entretanto, como a evolução homeopática era satisfatória esse tempo foi respeitado sem interferência desnecessária para ace-lerar a cura, já que o paciente permaneceu assintomático.

Embora todos os casos continuassem com hiperemia conjuntival e erosões puntiformes na córnea em $\mathrm{AO}$ por um certo tempo, a aceitabilidade das famílias das crianças nesse período foi boa, provavelmente relacionada com o alívio (e consequente menor interferência da ceratoconjuntivite primaveril nas atividades diárias dos enfermos) e, também, com a retirada da medicação convencional.

Segundo a homeopatia, quando há uma melhora da doença por um período significativo e depois os sintomas da enfermidade parecem retornar, muitas vezes, isso não interfere na cura e os sintomas desaparecem após poucos dias sem nova prescrição, proporcionando uma cura mais fácil, rápida e segura da totalidade da doença. Essas crises, com o passar do tempo, se reduzem tanto em frequência como em intensidade, e não devem sofrer interferências medicamentosas. ${ }^{6,7}$ Entretanto, é importante manter um acompanhamento regular do quadro oftalmológico até que os sinais e sintomas desapareçam.

Sob três aspectos a medicina homeopática parece ser alternativa terapêutica economicamente vigorosa para o tratamento da úlcera de córnea em escudo por ceratoconjuntivite primaveril. Nos nove casos o custo medicamentoso homeopático total foi muito inferior à estimativa de custo medicamentoso convencional, mensal e médio. Ela, ainda, não desencadeou efeitos adversos exigentes de cuidados específicos, responsáveis por encarecer a atenção à saúde. Além do mais, economizou gastos porque incluiu cuidados com co-morbidades por sua aptidão para abordar a totalidade orgânica enferma, sem se circunscrever a combater o mal particular. 18
Com o projeto mecanicista da biociência, a beneficência médica ampliou suas possibilidades desde os domínios do início até o fim da vida biológica. Mas também deflagrou crescente compressão da natureza e do humano. A biomedicina se depara com graves distorções da justiça distributiva e com iniquidades relativamente ao acesso às tecnologias de alto custo associadas à ciência de doenças, implicada ainda com importantes dilemas éticos vinculados a seu experimentalismo e aos riscos de suas intervenções. ${ }^{12,14,15}$

Facilitando o equilíbrio entre beneficência e maleficência, decorrentes da objetivação da natureza e implicadas com o imediatismo nos resultados da medicina de doenças, a homeopatia se estabelece no âmbito da assimilação não maleficente da suavidade e da empatia. Fundamentando-se no reconhecimento singular da representação de doença, ela amplia as bases do consentimento, atualmente reduzidas ao termo de consentimento livre e esclarecido. Ela os estende ao marco da relação médico-paciente fraterna que inclui a sensação de doença do enfermo pela memória experimental do médico homeopata de sensação semelhante. Nesse sentido a medicina homeopática propende, de acordo com a regra áurea do amor semelhante, para a otimização do respeito devido à alteridade e à autonomia dos pacientes, evoluindo a filantropia hipocrática. 19,20

Assim, a homeopatia parece colaborar para o diálogo entre os domínios ético e técnico, com vistas a otimizar a realização pacífica da vida, como expressão médica da regência semelhante da justiça (regra áurea) e, radicando-se em contexto não maleficente, parece encerrar consistentes contributos para a minoração de severas injustiças e iniquidades relativas ao manejo público e particular de recursos e de possibilidades favorecedoras da realização do humano. ${ }^{18}$ Ela se inclina, também, para o enriquecimento da reflexão e prática do consentimento e, assim, do respeito à autonomia. 19

A homeopatia pode contribuir para o tratamento de crianças portadoras de ceratoconjuntivite primaveril com úlcera de córnea em escudo, minimizando preocupações bioéticas com ele relacionadas, sendo que novas pesquisas são necessárias para melhor avaliar os resultados obtidos com este estudo.

\section{Considerações finais}

Com o advento da bioética, a justiça tem representado a abstração distributiva de bens e oportunidades decorrentes do progresso tecno-científico com vistas a auxiliar a realização da vida. ${ }^{21}$ Mas a justiça pode 
ser identificada nas raízes da cultura ocidental. Tanto ela quanto a não-maleficência. 22 Essas diretrizes éticas integram a regra áurea do amor semelhante que recomenda a orientação das relações (justiça) pela amizade, pela assimilação (princípio de similitude), já afirmada mítico-religiosamente como referencial de criação do humano (Homo como semelhante). ${ }^{19}$

A similitude presidiu curas em relatos de Homero e foi considerada por Hesíodo na promoção do comedimento para saneamento das aflições humanas. Posteriormente, filósofos naturalistas gregos a valorizaram em suas concepções cosmogônicas e o hipocratismo a situou no marco da amizade que mesclava desarmonias da isonomia das potências para temperar humores dominantes responsáveis pelas enfermidades.19,20,23-27 Paracelsus fundamentou-se nela ao conceber seu sistema de signaturas.

No entanto, foi com Hahnemann que se chegou a compreender o processo de manejo médico do princípio de semelhança. Fundamentado na experiência, ele entendeu que, para se tratar um enfermo segundo a natureza, é necessária pequeníssima dose de medicamento que produza, quando suavemente experimentado na saúde, perturbações assemelhadas às que o doente apresenta.6,7 Para ele, os provadores de eleição deviam ser os próprios médicos e as provas, auto-experimentações. 6

Pode-se reduzir os fundamentos da medicina homeopática ao princípio de similitude. 8 Destarte, ele é o referencial epistemológico da homeopatia. Seu manejo, mediante utilização da memória sintética experimental, enseja natural vivência do

\section{Referências}

1. Sena CM, Tanure MA, Cruz ACG, Trindade F, Pereira FAZ. Uso da medicação homeopática no tratamento da ceratoconjuntivite primaveril - resultados iniciais. Arq Bras Oftalmol. 2003; 66: 45-50

2. Sacchetti M, Baiardini I, Lambiase A, Aroni S, Fassio O, Gramiccioni C, Bonini S, Bonini S. Development and testing of the quality of life in children with vernal keratoconjunctivis questionnaire. Am J Ophthalmol. 2007; 144: 557-63.

3. Corum I,Yeniad B, Bilgin LK, Ilhan R. Efficiency of olopatadine hydrochloride $0,1 \%$ in the treatment of vernal keratoconjunctivitis and goblet cell density. J Ocular Pharmacol Ther. 2005; 21: 400-5

4. Goulart DA, Goulart DG, Cypel MC, Dantas PEC, Dantas MCN. Perfil clínico-epidemiológico de pacientes do ambulatório de alergia ocular da Santa Casa de São Paulo. Arq Bras Oftalmol. 2003; 66: 609-15.

5. Sacchetti M, Lambiase A, Mantelli F, Deligianni V, consenso, 10 consoante uma espécie de recordação, de acordo com preceitos hipocráticos. 23,27 Por este processo, a representação sintomática de doença é reconhecida pelo médico. Através dele, por sua vez, o paciente reconhece sentido para se re-equilibrar. Harmoniosamente, tal consenso evolui o consentimento (razão de ser do termo de consentimento livre e esclarecido), garantia indispensável aos cuidados com a autonomia nas relações médicas. ${ }^{28}$ Sentimentos (feridas de sentido) do médico e do enfermo se assemelham (assimilação) por uma atividade de memória firmada na recordação do médico. Esta assimilação traduz o necessário para que a temperança orgânica (saúde) se desenvolva. 19

As atividades homeopáticas, experimentais e terapêuticas, propendem à suavidade já que: a) a realização de suas provas no marco da saúde de voluntários conscientes, não no da vulnerabilidade do doente, 14,15,29 pelo uso, em mínima quantidade porque infinitesimalmente diluída, de medicamento único; b) por tender a tratar com baixo custo e mais não-maleficência, inclinando-se a ser muito bem aceita pelos pacientes $9,15,30,31 \mathrm{e}, \mathrm{c})$ por contribuir, também, para aperfeiçoar a cultura de paz, já que, no manejo médico do princípio de semelhança, é indispensável que haja uma ordenação de consenso natural na relação médico-paciente. 10 A regência de consenso é expressão da similitude que enseja o reconhecimento médico da representação de doença, bem como a escolha do medicamento, promovendo sentido e diálogo no âmbito da articulação médicopaciente, ampliando as possibilidades de satisfação da alteridade e implicando-se com a fundamentação da ética médica pela não-maleficência.19,22
Leonardi A, Bonini S. Tailored approach to the treatment of vernal keratoconjunctivitis. Ophthalmol. 2010; 117: 12949

6. Hahnemann S. Organon da arte de curar. Trad. Villela EM, Soares IC. Ribeirão Preto: Robe Editorial; 1996.

7. Hahnemann S. Doenças Crônicas. 2 ed. alemã. São Paulo: G.E.H Benoit Mure; 1984

8. Cruz ACG. Da substituibilidade em autopatogenesias que implica provadores e a propriedade medicinal por representação psíquica [Memória]. Minas Gerais: Serviço Phýsis de Homeopatia do Instituto Mineiro de Homeopatia; 2007. Disponível em: http://www.physishomeopatia.com.br/

9. Zulian Teixeira MZ. Ensaio clínico quali-quantitativo para avaliar a eficácia e a efetividade do tratamento homeopático individualizado na rinite alérgica perene [tese]. São Paulo: Faculdade de Medicina da Universidade de São Paulo; 2009. Disponível em: http://www.teses.usp.br/

10. Cruz ACG, Iannotti GC, Gouveia KFC, Beier M. A cultura 
homeopática de paz na saúde. Rev Med Minas Gerais 2007; 17: 303-9.

11. CFM (Conselho Federal de Medicina). Acrescentar na relação de especialidades reconhecidas pelo CFM, para efeito de registro de qualificação de especialistas a hansenologia e a homeopatia. Resolução n. 1.000/1980, de 21 Julho 1980. Lex: Diário Oficial da União. Seção I, Parte II.

12. Silva FL. Notas sobre a relação entre técnica e ética. Bioética. 2003; 11: 177-81.

13. Durand G. Introdução geral a bioética, história, conceitos e instrumentos. São Paulo: Loyola; 2007.

14. Junges JR. 1999. Bioética, perspectivas e desafios. São Leopoldo: Unisinos; 1999. p. 41-7.

15. Luz MT. Natural, racional, social: razão médica e racionalidade científica moderna. São Paulo: Hucitec; 2004. p. 4550 .

16. Thompson EA, Shaw A, Nichol J, Hollinghurst S, Henderson AJ, Thompson T, Sharp D. The feasibility of a pragmatic randomised controlled trial to compare usual care with usual care plus individualised homeopathy, in children requiring secondary care for asthma. Homeopathy. 2011; 100: 122-30

17. Vijnovsky B. Tratado de Matéria Médica Homeopática. 2 ed. São Paulo: Gráfica Editora Ltda; 1989. v. I,II,III.

18. Cruz ACG, Sena CM, Tanure MAG, Trindade F, Boteon JE. Melhora das papilas gigantes na ceratoconjuntivite primaveril com o uso de medicamento homeopático: dois casos. Rev Homeopatia. 2011; 74: 17-24.

19. Cruz ACG, Beier M. Consentimento e similitude: a autoexperimentação promovendo o diálogo entre a ética e a técnica. In: Salles AA. Bioética, velhas barreiras, novas fronteiras. Belo Horizonte: Mazza Edições; 2011. p. 15994.

20. Entralgo PL. La Medicina hipocrática. Madrid: Alianza Universidad; 1987.

21. Dallari SG. A bioética e a saúde pública. In: Costa SIF, Oselka G, Garrafa V, org. Iniciação à Bioética. Brasília: CFM; 1998. p. 205-16.

Recebido em 24 de maio de 2011

Versão final apresentada em 27 de agosto de 2012

Aprovado em 21 de setembro de 2012
22. Gracia D. Primum non nocere. El principio de no-maleficencia como fundamento de la ética médica. Madrid: Anzos S.A; 1990.

23. Hippocrates. Sobre la medicina antigua. In: Gual CG, editor. Tratados hipocráticos. Traduccion Nava MDL. Madrid: Editorial Gredos; 1983. v. I. p. 135-68.

24. Hippocrates. Sobre los lugares em el hombre. In: Gual CG, editor. Tratados hipocráticos. Traduccion Polo JV. Madrid: Editorial Gredos; 2003. v.VIII, p. 89-136.

25. Hippocrates. Epidemias VI. In: Gual CG, editor. Tratados hipocráticos. Traduccion Novo EG. Madrid: Editorial Gredos; 1989. v. V. p. 211-50.

26. Hippocrates. Sobre la enfermedad Sagrada. In: Gual CG, editor. Tratados hipocráticos. Traduccion Gual CG. Madrid: Editorial Gredos; 1983. v. I. p. 401-22.

27. Hippocrates. Preceptos. In: Gual CG, editor. Tratados hipocráticos. Traduccion Ferez JAL. Madrid: Editorial Gredos; 1983. v. I. p. 311-20.

28. Goldim JR, Clotet J, Francisconi CF. Consentimento informado e a sua prática na assistência e pesquisa no Brasil. Porto Alegre: Edipucrs; 2000.

29. Priven SIW. A experimentação de medicamentos no século XVIII. In: O saber fazer e seus muitos saberes: experimentos, experiências e experimentações. São Paulo: Livraria da física-EDUC-Fapesp; 2006. p. 314-24.

30. Marino R. Homeopathy and collective health: the case of dengue epidemics. Int J High Dilution Res. 2008; 7: 17985 .

31. Iannotti GC. Del impacto de la especialidad médica en la atención primaria de la salud en Belo Horizonte, Brasil. Especialistas en salud de la família, especialistas en Homeopatia [tese]. Argentina: Facultad de Ciências Médicas de la Universidad Nacional de Córdoba; 2008. 